Ngozi Ekeke', Elias Aniwada², Joseph Chukwu', Charles Nwafor', Anthony Meka', Chukwuka Alphonsus', Okechukwu Ezeakile', Adeyemi Ajayi ${ }^{3}$, Festus Soyinka ${ }^{4}$, Francis Bakpa ${ }^{5}$, Victoria Uwanuruochi ${ }^{6}$, Ezechukwu Aniekwensi', Chinwe Eze ${ }^{1}$

'German Leprosy and TB Relief Association, Nigeria

${ }^{2}$ University of Nigeria Teaching Hospital, Enugu, Nigeria

${ }^{3}$ Sacred Heart Hospital, Abeokuta, Nigeria

${ }^{4}$ Ogun State Ministry of Health, Abeokuta, Nigeria

${ }^{5}$ Delta State Ministry of Health, Nigeria

${ }^{6}$ Federal Medical Centre, Umuahia, Nigeria

${ }^{7}$ Federal Medical Centre, Asaba, Nigeria

\title{
Screening diabetes mellitus patients for tuberculosis in Southern Nigeria: A pilot study
}

\begin{abstract}
Introduction: Diabetes mellitus (DM) and tuberculosis (TB) are of great public health importance globally, especially in SubSaharan Africa. Tuberculosis is the third cause of death among subjects with non-communicable diseases. DM increases risk of progressing from latent to active tuberculosis. The study aimed to ascertain yield of TB cases and the number needed to screen (NNS) among DM patients.

Material and methods: A cross-sectional study was conducted at 10 health facilities with high DM patient load and readily accessible DOTS center in 6 states of southern region of Nigeria over a period of 6 months under routine programme conditions. All patients who gave consent were included in the study. Yield and NNS were calculated using an appropriate formula.

Results: 3457 patients were screened with a mean age (SD) of 59.9 (12.9) years. The majority were male, 2277 (65.9\%). Overall prevalence of TB was $0.8 \%$ (800 per 100000 ). Sixteen (0.5\%) were known TB cases (old cases). There were 221 presumptive cases (6.4\%) out of which 184 (83.3\%) were sent for Xpert MTB/Rif assay. Eleven (0.3\%) new cases of TB were detected, giving additional yield of $40.7 \%$ and the number needed to screen (NNS) of 315 . All the 11 patients were placed on anti-TB treatment. Conclusions: The prevalence of TB among DM patients was higher than in the general population. The yield was also good and comparable to other findings. This underscores the need for institute active screening for TB among DM patients. Further studies are recommended to identify associated factors to guide policy makers in planning and development of TB-DM integrated services.
\end{abstract}

Key words: diabetes, tuberculosis, screening, yield, number needed to be screened, Nigeria

Adv Respir Med. 2020; 88: 6-12

\section{Introduction}

Diabetes mellitus (DM) and tuberculosis (TB) are major lethal diseases of great public health importance globally [1]. This is the most visible in Sub-Saharan Africa (SSA) due to the converging epidemics of both communicable and non-communicable diseases. The World Health Organization (WHO) has identified DM as a global epidemic. The relationship between diabetes and tuberculosis as well as their synergistic role in causing human disease may be the next challenge for global tuberculosis control [2].

Diabetes prevalence is increasing globally, especially in low- and middle-income countries due to ageing, population growth, rapid economic, social, and lifestyle changes [3, 4]. About 422 million people worldwide were living with diabetes in 2014 [5]. The global prevalence of diabetes was estimated to be $8.5 \%$ among adults

Address for correspondence: Elias Aniwada, Department of Community Medicine, University of Nigeria Teaching Hospital, Enugu, Enugu State, Nigeria; e-mail: eaniwada@gmail.com DOI: 10.5603/ARM.2020.0072

Received: 18.08.2019

Copyright (C) 2020 PTChP

ISSN 2451-4934 
aged 18 years and above [5]. In 2012, diabetes was the direct cause of 1.5 million deaths [5]. Eighty percent (80\%) of these people live in lowand middle-income countries, including Nigeria where TB prevalence is equally high. This is also where $80 \%$ of all deaths due to DM occur [5]. About $10-15 \%$ of global TB cases are linked to diabetes [6]. Diabetes mellitus has recently emerged as risk factor for developing active TB [7]. Diabetes triples a person's risk of developing TB [8-11]. The global economic burden of diabetes is enormous. In Africa, the mean annual cost for diabetes care ranges between \$2 144 and \$11 430 (direct costs \$876-1 220) [12].

Tuberculosis ranks as the second leading cause of death from an infectious disease globally next to the human immunodeficiency virus (HIV). $\mathrm{DM}$ and TB comorbidities complicate tuberculosis management and negatively influence its treatment outcome [13, 14]. In 2014, 9.6 million people fell ill with TB, 1.5 million died from TB and one in three individuals in the world was infected with latent TB [5]. The World Health Organization (WHO) reported that in 2017, globally there were estimated 10.0 million incident cases of TB (range, 9.0-11.1 million), which translates to 133 cases (range, 120-148) per 100000 population. Most of these cases in 2017 occurred in Asia (44\%) and the African Region (24\%) [15]. In Nigeria, tuberculosis is still a grave public health problem. It is associated with huge economic burden just as in other low-income countries [16-18]. In Nigeria, despite the National TB and Leprosy Control Programme (NTBLCP) reporting 94604 cases in 2012, this number only represents 51\% of the cases estimated to have occurred in the country that year [19]. In 2017, the incidence of TB in Nigeria was 418 cases (range 273-594) [15].

The presence of DM alone does not justify screening or treatment of latent TB infection (LTBI). However, when combined with other risk factors for TB, the presence of DM may be sufficient to justify screening and treatment of LTBI, even in a low TB incidence setting [20]. The Sustainable Development Goals (SDGs) among other targets, made ending the global TB epidemic a priority. To achieve this, new strategies need to be employed. Passive case finding which used to be the norm whereby patients present themselves for TB screening seems not yielding desired result. Active case finding (ACF) is believed to contribute to the earlier detection of persons with TB. This will lead to early initiation of treatment, better therapy outcomes for individuals and ultimately, it will reduce transmission in the community [21-23].
The World Health Organization and the Union have launched a new 'Collaborative Framework for the care and control of Diabetes and Tuberculosis'with one of the most important activities being the routine implementation of bi-directional screening of the two diseases [24]. In Nigeria, there are no policies identifying DM patients with TB symptoms, or minimising the time spent by patients with probable DM-TB co-morbidity in the diabetes clinics. In addition, evidence to inform the development of interventions to address the TB burden among $\mathrm{DM}$ patients is crucial but lacking. Moreover, the methods of screening, recording and reporting DM and TB co-morbidity in routine health care settings are not well determined, and these knowledge gaps need to be addressed. There is a need for relevant stakeholders, including: WHO, National TB and Leprosy Control Programme and government agencies/departments responsible for non-communicable diseases to review and discuss linkages between DM and TB - hence the need for bidirectional screening and the WHO Union Collaborative Framework. A multicenter, unidirectional study to assess the burden of diabetes mellitus among TB patients was recently completed in 6 selected states in southern Nigeria the results of which have been published [25]. The study seeks to address the second arm, i.e. intensified TB screening among patients with diabetes mellitus. The feasibility of the screening (DM patients for TB), burden and challenges will be evaluated within routine health care services to inform policy change and develop generic protocols for its implementation.

Screening persons with DM for TB could be one of the strategies for early and increased TB case detection in Nigeria. These informed the programme implementation of active case finding through screening to ascertain incidence, yield, number needed to screen (NNS) for a case of TB among DM patients.

\section{Material and methods}

\section{Study area}

The study was conducted in the southern region of Nigeria spanning through all the 3 geopolitical zones and involving 6 states (two from each geopolitical zone) namely; Ogun, Ondo, Edo, Delta, Enugu and Abia States. Diabetes clinics of selected health facilities with high DM patient load and readily accessible DOTS centre were selected in each state for the study. This region hosts the major oil producing 
states. They engage in farming, fishing, mining, trading as well as civil/public services. There are well established health centres, including tertiary, secondary and primary facilities that care for patients, including those with DM and TB. However, 10 health clinics were used for the study. These were the major centres caring for both diseases in the states.

\section{Study design, duration and population}

A hospital-based cross-sectional study was done under programme implementation. The study was conducted over 8-month period from February to October 2018. All diagnosed cases of diabetes mellitus who were aged 15 years and above, registered with and attending Diabetes Clinics in selected health facilities within the study period and who gave informed consent were included.

\section{Sampling technique and sample size determination}

All diabetic clients attending DM clinics at the selected health facilities who met the inclusion criteria were included in the screening. They were recruited consecutively as they presented at clinic throughout the period of the study. A total of 3457 patients were included in the study.

\section{Procedure of screening}

The subjects were DM patients aged 15 years and above who have been registered in the DM clinic for care. Presumptive and diagnosed TB cases were screened for HIV in line with the national TB guidelines. The screening for TB was carried out at each patient's visit (minimum of one month interval; however, each subject was reported only once during the project period) using a symptom-based standardised checklist.

\section{Referral of identified presumptive TB cases (among DM patients) to TB clinic}

Trained research assistants worked in collaboration with the staff at the DM clinics to: conduct a symptomatic TB screening for all eligible DM patients using appropriate tools (checklist), identify presumptive TB cases among those screened for TB; collect 2 sputum specimens from identified presumptive TB cases; send sputum specimens for GeneXpert diagnosis (second sputum specimen was equally processed if the first was negative), refer all diagnosed TB cases to TB clinic for treatment and record necessary data using tools. Good cooperation and collaboration were established between the 2 sets of staff working in the different service areas (DM and TB clinics).

\section{Data collection and analysis}

Patient information was extracted from a standard globally used register and analysed. The records were filled by trained health workers to ensure good quality data. Double data entry was done to ensure accuracy. IBM Statistical Package for Social Sciences Version 21 was used for data entry, editing and analysis. Results were presented in tables. Yield [(new TB cases/total number with TB) $\times 100$ ] and the number needed to screen (number of patients/ /new TB cases) were calculated. Mean, standard deviation, proportion and percentages were used as summary measures, where appropriate.

\section{Ethical consideration}

The Ethics and Research Advisory Committee of University of Nigeria Teaching Hospital (UNTH), Enugu approved the study. Approval was also obtained from the State TB Control Programme in six states selected for the project. Permission was equally obtained from heads of the facilities and written informed consent obtained from the patients. Confidentiality of data was ensured in course of the research.

\section{Results}

Table 1 shows that a higher proportion of patients were aged $\geq 60$ years $2669(77.2 \%)$ and with their mean age 59.86 years. Males were higher in proportion 2277 (65.9\%). They were predominantly civil/public servants - 1428 (41.3\%) and traders - 1308 (37.8\%). The majority had BMI of $25-29.9 \mathrm{~kg} / \mathrm{m}^{2} 1340$ (38.8\%) followed by $\geq 30 \mathrm{~kg} / \mathrm{m}^{2} 1019$ (29.5\%). Only 22 (0.6\%) currently smoked cigarettes or tobacco-based products. As well, only $3(0.1 \%)$ tested positive for HIV. Most were type 2 DM 3328 (96.3\%).

Table 2 shows that 6386 patients attended the clinic within the 6 months of the study. Of this number, 3457 (54.1\%) were new patients or attended the clinic once. These were the patients further studies were based on. Presumptive cases were 221 (6.4\%) out of which $184(83.3 \%)$ were sent for Gene Xpert. In all $11(0.3 \%)$ tested positive following screening (new cases), $16(0.5 \%)$ were known cases (old cases), and 27 (0.8\%) had TB (old and new cases) among the patients studied. Among the 11 subjects that were newly detected, all were sent for treatment.

Table 3 shows that of the 3457 patients studied, yield was $40.7 \%$ and the number needed to 


\section{Table 1. Characteristics of patients}

\begin{tabular}{|c|c|c|}
\hline Variables & $\begin{array}{l}\text { Frequency } \\
(\mathrm{n}=3457)\end{array}$ & Percent (\%) \\
\hline \multicolumn{3}{|l|}{ Age (years) } \\
\hline$<60$ & 788 & 22.8 \\
\hline$\geq 60$ & 2669 & 77.2 \\
\hline Mean (SD) & $59.86(12.86)$ & \\
\hline \multicolumn{3}{|l|}{ Gender } \\
\hline Male & 2277 & 65.9 \\
\hline Female & 1180 & 34.1 \\
\hline \multicolumn{3}{|l|}{ Occupation } \\
\hline Civil/public servant & 1428 & 41.3 \\
\hline Trading/business & 1309 & 37.9 \\
\hline Others & 722 & 20.9 \\
\hline \multicolumn{3}{|l|}{ BMI } \\
\hline$<18.5$ & 102 & 3.0 \\
\hline $18.5-24.9$ & 996 & 28.7 \\
\hline $25-29.9$ & 1340 & 38.8 \\
\hline$\geq 30$ & 1019 & 29.5 \\
\hline \multicolumn{3}{|l|}{ Current smoker } \\
\hline Yes & 22 & 0.6 \\
\hline No & 3435 & 99.4 \\
\hline \multicolumn{3}{|l|}{ Type of DM } \\
\hline 1 & 129 & 3.7 \\
\hline 2 & 3328 & 96.3 \\
\hline \multicolumn{3}{|l|}{ HIV } \\
\hline Negative & 3454 & 99.9 \\
\hline Positive & 3 & 0.1 \\
\hline
\end{tabular}

screen (NNS) for the yield was 315 patients. When disaggregated by characteristics, those aged $<60$ years had yield of $42.9 \%$ and NNS of 263 , males had yield of $50.0 \%$ and NNS of 326 , civil/public servants had yield of $37.5 \%$ and NNS of 476 , those with BMI of $<18.5$ had yield of $60.0 \%$ and NNS of 34 , smokers had yield of $47.8 \%$ and NNS of 313 , type $2 \mathrm{DM}$ patients had yield of $55.6 \%$ and NNS of 333 and those that were negative for HIV had yield of $38.5 \%$ and NNS of 345 .

\section{Discussion}

The study reported that $11 \mathrm{DM}$ patients or $0.3 \%$ of them tested positive following screening (new cases). More findings were that yield of TB cases was $40.7 \%$ and the number needed to screen to make diagnosis of a TB case was $315 \mathrm{DM}$ patients. This is revealing and encouraging as these identified cases would have been the foci of spread among unsuspecting public with their consequent effects. The findings can partly be explained by the large population size involved in the study. It also gave credence to innovations aimed at controlling the menace of the diseases. For instance, the Collaborative Framework for the Care and Control of Diabetes and Tuberculosis as launched by WHO and the Union have routine implementation of bi-directional screening of the DM and TB as one of the important activities for control of TB [24]. In Nigeria where the methods of screening, recording and reporting DM and TB co-morbidity in routine health care settings are not well determined, these existing gaps can be addressed by such

Table 2. Distribution of patients studied

\begin{tabular}{lcc}
\hline Variables & Formula* & Value \\
\hline A. Total number of DM patient visits over 6 months (including revisit) & 6386 \\
B. Number (\%) of new DM patients studied (excluding revisit) & {$[(\mathrm{B} / \mathrm{A}) \times 100]$} & $3457(54.1)$ \\
C. Number (\%) of presumptive TB cases identified & {$[(\mathrm{C} / \mathrm{B}) \times 100]$} & $221(6.4)$ \\
D. Number (\%) sent for GeneXpert test & {$[(\mathrm{D} / \mathrm{C}) \times 100]$} & $184(83.3)$ \\
E. Number (\%) that with MTB detected (new cases) & {$[(\mathrm{E} / \mathrm{B}) \times 100]$} & $11(0.3)$ \\
F. Number placed on TB treatment & & 11 \\
G. Number (\%) of previously diagnosed (known) TB cases & {$[(\mathrm{E}+\mathrm{G})]$} & $16(0.5)$ \\
H. Total number (\%) of TB cases & $27(0.8)$ \\
\hline
\end{tabular}

*What was computed to get value

DM — diabetes mellitus; TB — tuberculosis; MTB — Mycobacterium tuberculosis 
Table 3. Distribution of patients yield and number needed to screen disaggregated by patient characteristics

\begin{tabular}{|c|c|c|c|c|c|c|}
\hline Variables & $\begin{array}{c}\text { Number } \\
\text { of patients }\end{array}$ & $\begin{array}{l}\text { Total number } \\
\text { with TB }\end{array}$ & $\begin{array}{c}\text { Known TB } \\
\text { cases }\end{array}$ & New TB cases & $\begin{array}{l}\text { Yield } \\
(\%)\end{array}$ & NNS \\
\hline & [A] & [B] & & [C] & {$[(C / B) \times 100]$} & {$[\mathrm{A} / \mathrm{C}]$} \\
\hline Overall & 3457 & 27 & 16 & 11 & 40.7 & 315 \\
\hline \multicolumn{7}{|l|}{ Age (years) } \\
\hline$<60$ & 788 & 7 & 4 & 3 & 42.9 & 263 \\
\hline$\geq 60$ & 2669 & 20 & 12 & 8 & 40.0 & 334 \\
\hline \multicolumn{7}{|l|}{ Gender } \\
\hline Female & 1180 & 13 & 9 & 4 & 30.8 & 295 \\
\hline Male & 2277 & 14 & 7 & 7 & 50.0 & 326 \\
\hline \multicolumn{7}{|l|}{ Occupation } \\
\hline Civil/public servant & 1426 & 8 & 5 & 3 & 37.5 & 476 \\
\hline Trading/business & 1309 & 13 & 9 & 4 & 30.8 & 328 \\
\hline Others & 722 & 6 & 2 & 4 & 66.7 & 181 \\
\hline \multicolumn{7}{|l|}{ BMI } \\
\hline$<18.5$ & 102 & 5 & 2 & 3 & 60.0 & 34 \\
\hline $18.5-24.9$ & 996 & 10 & 5 & 5 & 50.0 & 193 \\
\hline $25-29.9$ & 1340 & 6 & 4 & 2 & 30.3 & 670 \\
\hline$\geq 30$ & 1019 & 4 & 3 & 1 & 25.0 & 1019 \\
\hline \multicolumn{7}{|l|}{ Current smoker } \\
\hline No & 22 & 4 & 4 & 0 & 0.0 & - \\
\hline Yes & 3435 & 23 & 12 & 11 & 47.8 & 313 \\
\hline \multicolumn{7}{|l|}{ DM type (n) } \\
\hline 1 & 129 & 3 & 3 & 0 & 0 & - \\
\hline 2 & 3328 & 18 & 8 & 10 & 55.6 & 333 \\
\hline \multicolumn{7}{|l|}{ HIV status } \\
\hline Negative & 3454 & 26 & 16 & 10 & 38.5 & 345 \\
\hline Positive & 3 & 0 & 0 & 0 & 0 & - \\
\hline
\end{tabular}

$\mathrm{BMI}$ — body mass index; DM — diabetes mellitus; NNS — number needed to screen; HIV — human immunodeficiency virus; TB — tuberculosis

activities like this programme implementation screening exercise.

Above all, diagnosis of TB disease is the entry point in the management of TB cases. Consequently, early diagnosis through screening will lead to prompt commencement of chemotherapy; reduced transmission of the disease to unsuspecting populace they come in contact with and ultimately, a positive impact on the control of the disease in the general population.

Other previous studies supported findings from this study. In Taiwan, a screening done for pulmonary tuberculosis among the elderly with type 2 diabetes involving 3087 patients detected $7(0.2 \%)$ patients who were positive for pulmonary tuberculosis [26]. Similarly, in a symptom screening project in a tertiary care hospital in south India, 12 cases were newly diagnosed of TB among 125 subjects that underwent TB investigation [27]. Another symptom screening research in Mexico detected 38 TB cases in 7763 diabetes patients [28]. In China, TB was diagnosed in 14 of 4085 patients with DM following active screening $[29,30]$. Findings from previous studies have shown that screening for active TB among diabetics could improve case detection just like in other populations susceptible to TB, including HIV-infected individuals, gold miners, and prisoners in developing countries [31].

The number of diabetics needed to screen to find one extra case of TB is directly related to the TB prevalence among that population. For instance, in areas with TB prevalence less than 25 per 100000 persons, at least 1000 diabetic 
individuals have to be screened to find one extra case of TB. With increasing prevalence, the number needed to screen to find one additional case of TB ranges from 4 to 442 . This implies that the yield of screening increases with the prevalence of TB in that region [31]. Also, the population attributed risk for TB from DM is dependent upon DM prevalence as evidenced in studies which documented that in populations with a higher incidence of $\mathrm{TB}, \mathrm{DM}$ is a more important risk factor [32]. DM accounts for a small proportion of TB cases in settings such as Australia with a low incidence of TB [33]. This number was $14.8 \%$ in India and $25 \%$ in a Mexican setting due to higher incidence and prevalence [34].

None of those that tested positive for HIV had TB. This is good as it would be disastrous for a patient to have two recognized major threats to TB diagnosis and treatment outcome. This may be explained by the age distribution of the studied DM patients. Most were aged $>60$ years of which most HIV positive subjects may not live as long as that due to either the comorbidities, drug-drug interactions or opportunistic infections as both DM and HIV infection reduce immunity. Human immunodeficiency virus infection is considered as the most potent risk factor for TB. Nevertheless, the high prevalence of DM in the world and its effect on TB burden is greater than that of HIV infection [7]. Even though HIV has been documented to be the strongest risk factor for TB at an individual level, DM is seen as most important at the population level. This indicates that having both will carry far-reaching health and economic implications.

\section{Conclusions}

The number of positive cases identified following screening, yield of TB cases and the number needed to screen to make diagnosis of a TB case were encouraging. Active case finding in the form of screening as carried out in this study is needed to end the global TB. Such programme implementation should be encouraged and advanced to reduce scourge of TB and its co-morbidities.

\section{Acknowledgement}

We are grateful to German Leprosy and Tuberculosis Relief Association for all their support in course of this research. We thank the management of University of Benin Teaching Hospital Benin, Federal Medical Centre Abeokuta, Federal Medical Centre Umuahia, Federal Medical Centre
Asaba, Enugu State University Teaching Hospital Enugu, University of Nigeria Teaching Hospital Enugu, University of Medical Science Teaching Hospitals Complex Akure, Sacred Heart Hospital, Lantoro, Abeokuta and Central Hospital, Warri for granting access to their institutions for data collection.

\section{Conflict of interest}

None declared.

\section{References:}

1. Syal K, Srinivasan A, Banerjee D. VDR, RXR, coronin-1 and interferon $\gamma$ levels in pbmcs of type-2 diabetes patients: molecular link between diabetes and tuberculosis. Indian J Clin Biochem. 2015; 30(3): 323-328, doi: 10.1007/s12291-014-0431-7, indexed in Pubmed: 26089620.

2. Ottmani SE, Murray MB, Jeon CY, et al. Consultation meeting on tuberculosis and diabetes mellitus: meeting summary and recommendations. Int J Tuberc Lung Dis. 2010; 14(12): 15131517, indexed in Pubmed: 21180207.

3. Harries AD, Lin Y, Satyanarayana S, et al. The looming epidemic of diabetes-associated tuberculosis: learning lessons from HIV-associated tuberculosis. Int J Tuberc Lung Dis. 2011; 15(11): 1436-44, i, doi: 10.5588/ijtld.11.0503, indexed in Pubmed: 21902876 .

4. International Diabetes Federation. IDF diabetes atlas. 4th edition. Brussels 2009.

5. World Health Organisation. Tuberculosis and Diabetes. Available at: www.who.int/tb (Accessed 12.02.2019).

6. Harries AD, Lin Y, Satyanarayana S, et al. The looming epidemic of diabetes-associated tuberculosis: learning lessons from HIV-associated tuberculosis. Int J Tuberc Lung Dis. 2011; 15(11): 1436-44, i, doi: 10.5588/ijtld.11.0503, indexed in Pubmed: 21902876.

7. Lai RPJ, Nakiwala JK, Meintjes G, et al. The immunopathogenesis of the HIV tuberculosis immune reconstitution inflammatory syndrome. Eur J Immunol. 2013; 43(8): 1995-2002, doi: 10.1002/eji.201343632, indexed in Pubmed: 23928963.

8. Pablos-Méndez A, Blustein J, Knirsch CA. The role of diabetes mellitus in the higher prevalence of tuberculosis among Hispanics. Am J Public Health. 1997; 87(4): 574-579, doi: 10.2105/ ajph.87.4.574, indexed in Pubmed: 9146434.

9. Restrepo BI. Convergence of the tuberculosis and diabetes epidemics: renewal of old acquaintances. Clin Infect Dis. 2007; 45(4): 436-438, doi: 10.1086/519939, indexed in Pubmed: 17638190 .

10. Ruslami R, Aarnoutse RE, Alisjahbana B, et al. Implications of the global increase of diabetes for tuberculosis control and patient care. Trop Med Int Health. 2010; 15(11): 1289-1299, doi: 10.1111/j.1365-3156.2010.02625.x, indexed in Pubmed: 20955495.

11. Jeon CY, Murray MB. Diabetes mellitus increases the risk of active tuberculosis: a systematic review of 13 observational studies. PLoS Med. 2008; 5(7): e152, doi: 10.1371/journal. pmed.0050152, indexed in Pubmed: 18630984.

12. Kirigia JM, Sambo HB, Sambo LG, et al. Economic burden of diabetes mellitus in the WHO African region. BMC Int Health Hum Rights. 2009; 9: 6, doi: 10.1186/1472-698X-9-6, indexed in Pubmed: 19335903.

13. Dooley KE, Chaisson RE. Tuberculosis and diabetes mellitus: convergence of two epidemics. Lancet Infect Dis. 2009; 9(12): 737-746, doi: 10.1016/S1473-3099(09)70282-8, indexed in Pubmed: 19926034.

14. Wang CS, Yang CJ, Chen HC, et al. Impact of type 2 diabetes on manifestations and treatment outcome of pulmonary tuberculosis. Epidemiol Infect. 2009; 137(2): 203-210, doi: 10.1017/ S0950268808000782, indexed in Pubmed: 18559125.

15. World Health Organization. Global Tuberculosis Report 2018. 16. World Health Organization. Global tuberculosis control 2015. 
17. Chaisson RE, Martinson NA. Tuberculosis in Africa - combating an HIV-driven crisis. N Engl J Med. 2008; 358(11): 1089-1092, doi: 10.1056/NEJMp0800809, indexed in Pubmed: 18337598 .

18. World Health Organization. Global tuberculosis control: epidemiology, strategy, financing. 2009.

19. Abebe M, Doherty M, Wassie L, et al. TB case detection: can we remain passive while the process is active? Pan Afr Med J. 2012; 11: 50, indexed in Pubmed: 22593786.

20. Dobler CC, Flack JR, Marks GB. Risk of tuberculosis among people with diabetes mellitus: an Australian nationwide cohort study. BMJ Open. 2012; 2(1): e000666, doi: 10.1136/ bmjopen-2011-000666, indexed in Pubmed: 22331390.

21. Raviglione M, Marais B, Floyd K, et al. Scaling up interventions to achieve global tuberculosis control: progress and new developments. Lancet. 2012; 379(9829): 1902-1913, doi: 10.1016 S0140-6736(12)60727-2, indexed in Pubmed: 22608339.

22. Lönnroth K, Castro KG, Chakaya JM, et al. Tuberculosis control and elimination 2010-50: cure, care, and social development. Lancet. 2010; 375(9728): 1814-1829, doi: 10.1016/S01406736(10)60483-7, indexed in Pubmed: 20488524.

23. Van't Hoog AH, Marston BJ, Ayisi JG, et al. High prevalence of pulmonary tuberculosis and inadequate case finding in rural western Kenya. Am J Respir Crit Care Med. 2011; 183(9): 1245-1253, doi: 10.1164/rccm.201008-12690C, indexed in Pubmed: 21239690.

24. World Health Organization and the International Union Against Tuberculosis and Lung Disease. Collaborative Framework for Care and Control of Tuberculosis and Diabetes. WHO/HTM/ TB/2011.15. Geneva, Switzerland: WHO, 2011.

25. Ekeke N, Ukwaja KN, Chukwu JN, et al. Screening for diabetes mellitus among tuberculosis patients in Southern Nigeria: a multi-centre implementation study under programme settings. Sci Rep. 2017; 7: 44205, doi: 10.1038/srep44205, indexed in Pubmed: 28281682

26. Baghaei P, Marjani M, Javanmard P, et al. Diabetes mellitus and tuberculosis facts and controversies. J Diabetes Metab
Disord. 2013; 12(1): 58, doi: 10.1186/2251-6581-12-58, indexed in Pubmed: 24360398.

27. Wild S, Roglic G, Green A, et al. Global prevalence of diabetes: estimates for the year 2000 and projections for 2030. Diabetes Care. 2004; 27(5): 1047-1053, doi: 10.2337/diacare.27.5.1047, indexed in Pubmed: 15111519.

28. Goldhaber-Fiebert JD, Jeon CY, Cohen T, et al. Diabetes mellitus and tuberculosis in countries with high tuberculosis burdens: individual risks and social determinants. Int J Epidemiol. 2011; 40(2): 417-428, doi: 10.1093/ije/dyq238, indexed in Pubmed: 21252210.

29. Ruslami R, Aarnoutse RE, Alisjahbana B, et al. Implications of the global increase of diabetes for tuberculosis control and patient care. Trop Med Int Health. 2010; 15(11): 1289-1299, doi: 10.1111/j.1365-3156.2010.02625.x, indexed in Pubmed: 20955495.

30. Singla R, Khan N, Al-Sharif N, et al. Influence of diabetes on manifestations and treatment outcome of pulmonary TB patients. Int J Tuberc Lung Dis. 2006; 10(1): 74-79, indexed in Pubmed: 16466041.

31. Jeon CY, Harries AD, Baker MA, et al. Bi-directional screening for tuberculosis and diabetes: a systematic review. Trop Med Int Health. 2010; 15(11): 1300-1314, doi: 10.1111/j. 1365-3156.2010.02632.x, indexed in Pubmed: 20958887.

32. Stevenson CR, Forouhi NG, Roglic G, et al. Diabetes and tuberculosis: the impact of the diabetes epidemic on tuberculosis incidence. BMC Public Health. 2007; 7: 234, doi: 10.1186/14712458-7-234, indexed in Pubmed: 17822539.

33. Raviglione M, Marais B, Floyd K, et al. Scaling up interventions to achieve global tuberculosis control: progress and new developments. Lancet. 2012; 379(9829): 1902-1913, doi: 10.1016/ S0140-6736(12)60727-2, indexed in Pubmed: 22608339.

34. Young F, Critchley JA, Johnstone LK, et al. A review of co-morbidity between infectious and chronic disease in Sub Saharan Africa: TB and diabetes mellitus, HIV and metabolic syndrome, and the impact of globalization. Global Health. 2009; 5: 9 doi: 10.1186/1744-8603-5-9, indexed in Pubmed: 19751503. 